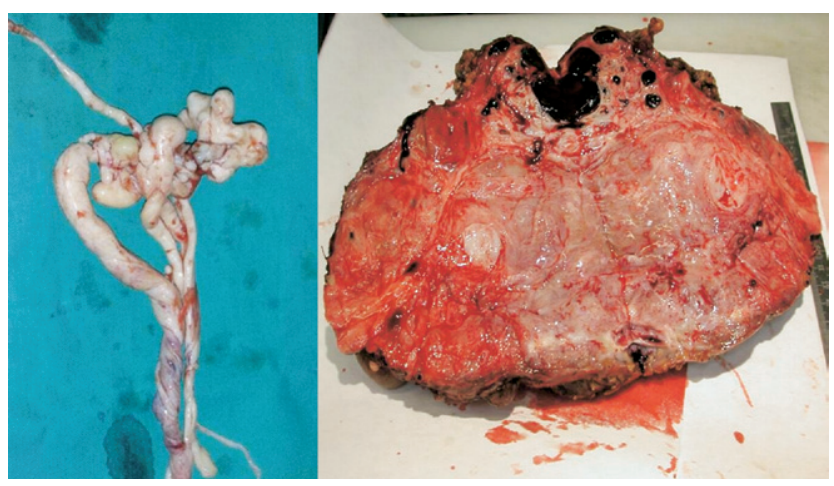

Figure 2. Intraoperative specimen. Intravenous leiomyomatosis extending into the right side of the heart, taking the shape of the inferior vena cava and right cavities (left). Abdominal tumor with abnormal vascular structures (right).

complete surgical tumor excision because recurrence is frequent despite the benign nature of the neoplasm, removal of ovarian function, and avoidance of postoperative estrogenic replacement therapy. ${ }^{1}$ Different surgical techniques have been proposed to approach intracardiac and abdominal neoplasms in the same or in separate operations. ${ }^{2}$ The detection of cytoplasmic estradiol and progesterone receptors in both the intravascular tumor and the metastasizing leiomyoma justify the use of an anti-estrogen, such as Tamoxifen, to control the disease progress. ${ }^{2}$ Because of the elevated incidence of recurrence $(30 \%)$ and the low metastatic potentiality, 3- and 6-month follow-ups after surgery are necessary. ${ }^{3}$

We stress the necessity of a pre-postoperative multidisciplinary approach to intravenous leiomyomatosis. We performed tumoral resection with a double-staged surgical strategy of cardiovascular and gynecologic operations to expose the patient to a lower postoperative bleeding risk. Hormonal therapy with Tamoxifen seems to be justified.

We thank Dr Tamburino Corrado, from the Department of Cardiology, Ospedale Ferrarotto, Catania, Italy; Dr Mignosa Carmelo, from the Department of Cardiosurgery, Ospedale S. Vincenzo, Taormina, Messina, Italy; and Drs Virzì Rosanna and Villari Loredana, from the Department of Pathology, Ospedale Vittorio Emanuele, Catania, Italy.

\section{References}

1. Steinmetz OK, Bedard P, Prefontaine ME, et al. Uterine tumor in the heart: intravenous leiomyomatosis. Surgery. 1996;119:226-9.

2. Lo KW-K, Lau T-K. Intracardiac leiomyomatosis. Case report and literature review. Arch Gynecol Obstet. 2001;264:209-10.

3. Evans AT 3rd, Symmonds RE, Gaffey TA. Recurrent pelvic intravenous leiomyomatosis. Obstet Gynecol. 1981;57:260-4.

\title{
A different surgical approach for an intrathoracic expanding hematoma
}

Kalliopi Athanassiadi, MD, ${ }^{a}$ Hans-Peter Reiffen, MD, PhD, ${ }^{\mathrm{b}}$ Nicolas Dickgreber, MD, PhD, ${ }^{\mathrm{c}}$ Florian Laenger, MD, PhD, ${ }^{\mathrm{d}}$ Christa-Maria Eschenbruch, MD, PhD, ${ }^{\mathrm{c}}$ Matthias Wilchelmi, MD, PhD, ${ }^{a}$ and Axel Haverich, MD, PhD, ${ }^{a}$ Hannover, Germany

hronic expanding hematomas are a rare clinical entity mimicking invasive soft-tissue neoplasms. We report the successful surgical treatment of a rare case of chronic expanding hematoma in the pleural cavity of an 83-year-old patient with a massive hemoptysis who was medically treated for tuberculosis 55 years earlier. A dissection of the

From the Departments of Thoracic and Cardiovascular Surgery, ${ }^{a}$ Anaesthesiology, ${ }^{\mathrm{b}}$ Pulmonology, ${ }^{\mathrm{c}}$ and Pathology, ${ }^{\mathrm{d}}$ Medical School of Hannover, Hannover, Germany.

Received for publication Sept 19, 2006; accepted for publication Oct 23, 2006.

Address for reprints: Kalliopi Athanassiadi, MD, Department for Thoracic and Cardiovascular Surgery, Medical School of Hannover, Carl-Neuberg Str. 1, 30625 Hannover, Germany (E-mail: kallatha@otenet.gr).

J Thorac Cardiovasc Surg 2007;133:832-4

$0022-5223 / \$ 32.00$

Copyright $\odot 2007$ by The American Association for Thoracic Surgery doi:10.1016/j.jtcvs.2006.10.049 calcified mass and extirpation of the hematoma were performed, the whole lung was expanded, the lobar and segmental arteries and bronchi were dissected, and the bronchial arteries were cauterized. In a follow-up of 4 months, the patient presented no recurrence, and a major operation (eg, a pleuropneumonectomy) was not necessary.

Chronic expanding hematomas are a rare clinical entity mimicking invasive soft-tissue neoplasms and producing mediastinal compression, usually in patients with a history of surgery for tuberculosis or tuberculous pleurisy. ${ }^{1,2}$

We report the successful surgical treatment of a rare case of chronic expanding hematoma in the pleural cavity of a patient with a massive hemoptysis who was medically treated for tuberculosis 55 years earlier.

\section{Clinical Summary}

An 83-year-old man was admitted to our department with hemoptysis and chest discomfort. He had a free medical history with the exception of tuberculosis 55 years ago. Chest radiography showed 


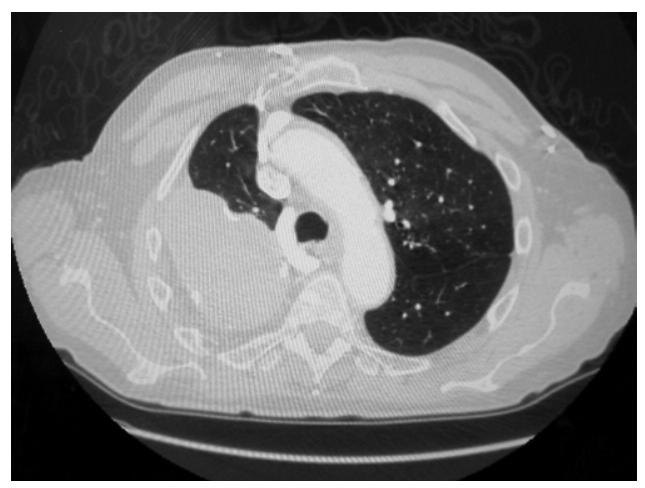

Figure 1. Chest computed tomography: a right intrathoracic mass with a calcified wall compressing the right lung to the mediastinum.

a complete opacification of the right hemithorax, and computed tomography revealed a well-circumscribed encapsulated lesion consisting of a thick calcified wall expanding to the whole pleural cavity and causing mediastinal compression (Figure 1). Bronchoscopy demonstrated arterial bleeding from the right lower lobe, and drug therapy including the topical use of adrenaline was initiated. An angiography performed for embolization could not identify the source of bleeding. Because of recurrent episodes and a massive hemoptysis within 6 days of hospitalization, emergency surgery was performed. The patient underwent a right lateral thoracotomy and a laterodorsal dissection of the calcified mass (Figure 2). The calcified wall was found to be adherent to the underlying lung but was easily dissected from the pleura parietalis. With a puncture, a cystic mass was identified and carefully opened. Extirpation of the hematoma followed (Figure 3), and the whole lung was mobilized from the mediastinum to the pleural cavity. The lobar and segmental arteries and bronchi were dissected, and cauterization of the bronchial arteries was performed. The anesthesiologist fully expanded the lung so that the remaining walls fell together.

Histology demonstrated a capsule consisting of hyalinized, hypocellular fibrous tissue. The material in the cavity consisted of

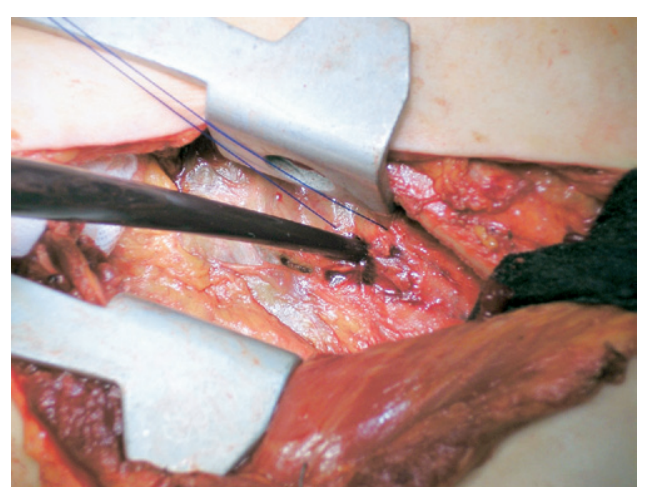

Figure 2. Intraoperative picture of the calcified cyst.

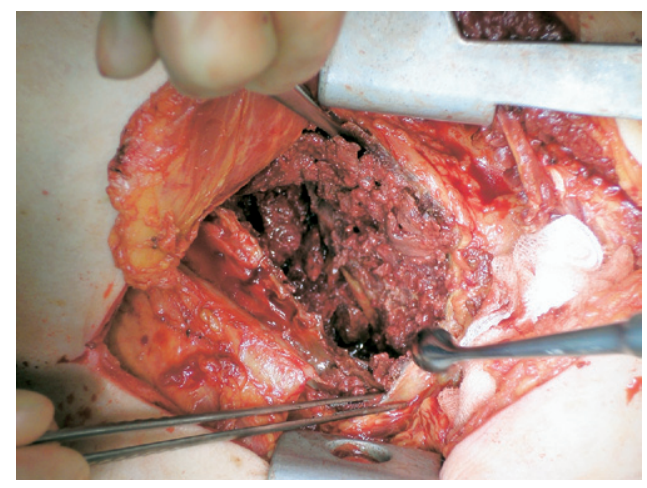

Figure 3. Intraoperative opening of the cavity revealed a wellcircumscribed organized hematoma.

dense collagenous tissue, many small eosinophilic amorphous necrotic debris, and red blood cells surrounded by xanthogranulomatous foreign body reactions (Figure 4). Cholesterin deposition was also found within the xanthogranulomatous reactive tissue. In addition, calcification on the outer wall was observed. No bacteria, including mycobacteria, were detected in the lesion.

The patient remained intubated for 48 hours in the intensive care unit. After a complicated postoperative course with bleeding, the patient was discharged 4 weeks later and presented no complications for the next 4 months.

\section{Discussion}

Hematomas caused by surgery or trauma usually resolve without sequelae. ${ }^{1}$ However, there have been a few cases of expanding hematomas, even over a period of 30 years or more, after medical or surgical treatment of tuberculosis. These cases are found more frequently in the Japanese literature ${ }^{1-4}$ than in the European or American literature.

The pathogenesis is poorly understood. Labadie and Glover ${ }^{5}$ demonstrated that the breakdown of leucocytes, hemoglobin, platelets, and

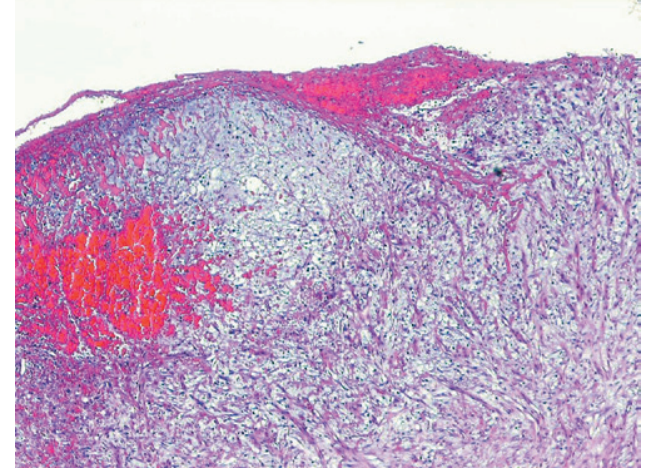

Figure 4. Histology: The inner aspect of the organizing hematoma with a loose granulation-tissue like proliferation of fibroblasts with numerous interspersed erythrocytes. A fibrinous exudate can be seen on the surface. 
fibrin results in an inflammatory process that effectively damages the capillaries of a capsule, increasing the permeability of the vascular wall and producing bleeding from dilated microvessels beneath the fibrous capsule. In some cases, erosion of microvessels may cause massive hemoptysis. Despite the calcification of the capsule, it seems that a small degree of elasticity exists, which is the reason hematomas usually expand over a long period of time.

These lesions are difficult to differentiate from soft-tissue sarcomas or other malignancies because many of them also reveal hemorrhagic and cystic changes on radiology. ${ }^{2-4}$ The best diagnostic tools are computed tomography and magnetic resonance imaging, including guided fine-needle biopsy.

Hanagiri and colleagues ${ }^{4}$ reported that palliative procedures, such as removal of the inner substance without whole capsule excision, may produce uncontrollable bleeding or recurrence of the hematoma within several years. In cases such as the one reported, the authors believe that a complete excision would have only been possible with a pleuropneumonectomy, which the 83-year-old patient would have not survived. The careful removal of the hematoma along with the mobilization and a partial decortication of the lung, dissection and control of all lobar pulmonary arteries and bronchi, cauterization of the bronchial arteries, and full reex- pansion of the lung with a longer intubation period and an intensive physiotherapy immediately postoperatively is the best solution in these elderly patients.

To our knowledge this is the first report of an elderly patient who was treated successfully for an intrathoracic expanding hematoma by cauterization of the bronchial arteries, an older method used in cases of pulmonary bronchiectasis.

\section{References}

1. Hirai S, Hamanaka Y, Mitsui N, Isaka M, Kobayashi T. Chronic expanding hematoma in the pericardial cavity after cardiac surgery. Ann Thorac Surg. 2003;75:1629-31.

2. Okubo K, Okamoto T, Isobe J, Ueno Y. Rupture of chronic expanding hematoma of the thorax into the lung parenchyma. J Thorac Cardiovasc Surg. 2004;127:1838-40.

3. Hwang GL, Moffatt SD, Mitchell JD, Leung AN. Chronic expanding hematomas of the thorax. AJR Am J Roentgenol. 2003;180:1182-3.

4. Hanagiri T, Murunaka H, Hashimoto M, Nishio T, Sakai S, Ono M, et al. Chronic expanding hematoma in the chest. Ann Thorac Surg. 1997;64:559-61.

5. Labadie EL, Glover D. Physiopathogenesis of subdural hematomas. Part I: Histological and biochemical comparisons of subcutaneous hematomas in rats with subdural hematoma in man. J Neurosurg. 1976 45:382-92.

\title{
Surgical exclusion of a thrombosed azygos vein aneurysm causing pulmonary embolism
}

\author{
Yoshitsugu Nakamura, MD, Kiyoharu Nakano, MD, Hayao Nakatani, MD, Takuya Fukuda, MD, Kentaro Honda, MD,
} and Nobuyuki Homma, MD, Tokyo, Japan

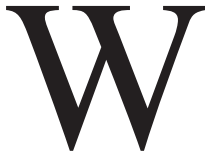

e report a surgical case of a large thrombosed azygos vein aneurysm causing severe pulmonary embolism. The aneurysm diminished spontaneously by closing its connection with the superior vena cava.

\section{Clinical Summary}

A 37-year-old woman was admitted with chest pain and palpitations. A superior vena caval aneurysm had been diagnosed 2 years ago during the patient's left thyroid lobe resection. Her medical

From the Department of Cardiovascular Surgery, Kanto Medical Center NTT EC, Tokyo, Japan.

Received for publication Oct 17, 2006; accepted for publication Nov 9, 2006.

Address for reprints: Yoshitsugu Nakamura, MD, Department of Cardiovascular Surgery, Kanto Medical Center NTT EC, 5-9-22 Higashigotanda, Shinagawa-ku, Tokyo 141-8625, Japan (E-mail: nakamura@kmc.mhc.east. ntt.co.jp).

J Thorac Cardiovasc Surg 2007;133:834-5

$0022-5223 / \$ 32.00$

Copyright $\odot 2007$ by The American Association for Thoracic Surgery

doi:10.1016/j.jtcvs.2006.11.012 history was unremarkable except for thyroid disease. Furthermore, no significant trauma was recorded. Her cardiac, renal, and hepatic functions were normal. A chest radiograph showed mediastinal enlargement occupying the right upper lung field (Figure 1, A). Computed tomography (CT) revealed a huge thrombosed saccular aneurysm of $11 \times 9 \mathrm{~cm}$ at the azygos vein arch (Figure $2, A$ ). The thrombus protruded into the superior vena cava (SVC), and bilateral massive pulmonary embolism was detected (Figure 2, B). Medical therapy for anticoagulation was not effective. Therefore, an emergency operation was performed. Through a median sternotomy, a cardiopulmonary bypass was established by right femoral and left brachiocephalic vein drainage cannulations and an ascending aortic return cannulation. The SVC was snared and intercepted at the junction to the right atrium and left brachiocephalic vein. The SVC was longitudinally incised. The orifice of the azygos vein was $2 \times 2 \mathrm{~cm}$ on the posterior wall of the SVC. The protruding thrombus was removed from the SVC. The orifice was closed with a 4-0 polypropylene running suture. The aneurysm itself was not removed. The right pulmonary artery was incised, and the embolectomy was completed. The postoperative course was uncomplicated. Four years after the operation, the shadow of the aneurysm disappeared on chest radiograph (Figure 1, B), and the aneurysm diminished to $15 \mathrm{~mm}$ in diameter on CT. 